\title{
Philosophiques
}

\section{Robert Hébert : L'Amérique française devant l'opinion étrangère 1756-1960. Anthologie, Montréal, Édtiions de l'Hexagone, 1989, 246 p.}

\section{André Vidricaire}

Volume 18, numéro 2, automne 1991

URI : https://id.erudit.org/iderudit/027157ar

DOI : https://doi.org/10.7202/027157ar

Aller au sommaire du numéro

Éditeur(s)

Société de philosophie du Québec

\section{ISSN}

0316-2923 (imprimé)

1492-1391 (numérique)

Découvrir la revue

Citer cet article

Vidricaire, A. (1991). Robert Hébert : L'Amérique française devant l'opinion

étrangère 1756-1960. Anthologie, Montréal, Édtiions de l'Hexagone, 1989, 246 p.

Philosophiques, 18(2), 159-169. https://doi.org/10.7202/027157ar d'utilisation que vous pouvez consulter en ligne.

https://apropos.erudit.org/fr/usagers/politique-dutilisation/ 


\section{ÉTUDES CRITIQUES}

ROBERT HÉBERT: L'Amérique française devant l'opinion étrangère 1756-1960. Anthologie, Montréal, Éditions de l'Hexagone, 1989, 246 p.

par André Vidricaire

Voici un livre "essentiel " à qui s'intéresse à l'histoire des idées et à la philosophie au nord du $45^{\mathrm{e}}$ parallèle. Dans cette anthologie de textes d'auteurs européens et américains qui ont écrit sur l'Amérique française, le lecteur d'ici et d'aujourd'hui se voit à travers et par les yeux de l'Autre. Mieux encore, dans la mesure où ces écrivains étrangers sont des "idéologues-cautions 》 pour les indigènes observés, le lecteur perçoit que ces images issues du regard de l'Autre, sont en même temps des miroirs des propres représentations que cette même Amérique française (les élites?) se faisait d'elle-même. Jeux d'images-miroirs en chassés-croisés où se confondent les projections de soi-même aux projections d'autrui sur soi.

Cette doxographie est précédée d'une présentation (pp. 7-24)* de la problématique accompagnée de "notes" (pp. 25-27) extrêmement pertinentes sur des notions comme "enclave ", " exotisme ", "querelle du Nouveau Monde ", " regard d'autrui ", etc. Enfin, elle se termine sur des "sources et filons bibliographiques" (pp. 215-245) qui constituent, au dire de Hébert, "l'armature essentielle" (p. 13) de son projet qui vise à offrir de nouvelles pistes de recherche. Somme toute, il ne s'agit que d'une anthologie parmi bien d'autres dont l'organisation est fort classique. Qu'à cela ne tienne: ce livre est à placer à côté du "livre anarcho-bibliographique » de Roland Houde (1980 et 1983e)", des dictionnaires, des répertoires et des grands ouvrages de référence sur le Québec. Ce compendium est un livre-programme, un livrechantier pour les penseurs d'ici.

Les pages renvoient au livre recensé.

" Les années renvoient aux repères bibliographiques. 


\section{Pour une recherche ethnophilosophique}

Jacques Beaudry dans La philosophie et le Québec. Des noms et des notes, Sherbrooke, 1989 (pp. 76 à 80), montre que Robert Hébert a un itinéraire intellectuel impressionnant. Voyez en annexe quelques références qui soulignent l'ampleur de sa démarche critique, généreuse et non hostile: elles peuvent inciter à (re)lire ce questionnement visionnaire. Notez que depuis les années 1973 il s'adonne à une vaste entreprise de recherche ethnophilosophique dans laquelle s'insère cet " essai bibliographique" (p. 8 et 1983e). Bref, ce livre-ci s'inscrit dans un contexte que je tenterai d'évoquer pour mieux saisir les enjeux et les buts. Aussi, mon propre texte devra se lire comme un collage qui assemble de multiples réflexions à méditer.

"Rechercheur» (1989) infatigable, Hébert a choisi de refaire à nouveau le long chemin oublié du "Je me souviens" philosophique. Butant sur les interprétations entendues et reçues, les poncifs, les lieux communs, les répétitions "idéogoniques", Hébert requestionne le "ce qui va de soi " tout comme le "ce qui est décrété bien " de penser et de citer (1984e). Faisant fi des spécialisations disséminées dans des tours d'ivoire givrées, il démultiplie les percées et les perspectives tout en cherchant à colmater la dispersion, réunir, rassembler, contaminer pour transformer ces savoirs et ouvrir un vaste champ de problèmes. Sur une terre d'idées labourée et toute tracée, Hébert propose de nouveaux sillons. Comme au temps passé, son ouvrage d'ethnophilosophe se fait à la main. Il cherche à remplacer certaines orthodoxies par une nouvelle orthopraxis sur une terre où le plus difficile est de tracer un premier sillon bien droit - plus vrai parce que collé à sa géographie - en symbiose avec les désirs / attentes du milieu. L'errance libertaire de ce "Canadien » est séminale à plusieurs points de vue.

En effet, depuis plus d'une quinzaine d'années, Hébert poursuit un double questionnement critique (1) sur notre rapport à la philosophie et (2) sur notre rapport à notre histoire (1983e). Il vise par la transformation de ces deux rapports à "déterritorialiser " notre propre espace de réflexion "post(néo-) colonial " (1983e) et endocolonial, méprisé, occulté et impensé. Donnant corps, épaisseur, chair, matière (grise) à cette notion de "lieu dit commun " qui renvoie à l'impensé de nos usages, Hébert assigne à la philosophie la tâche critique de transformer tout lieu commun dont "l'actualité souvent aveuglante [...] nous opprime »(1984a), par une analyse philosophico-historique de ces déterminations (1983a). Ainsi, partant à la découverte de l'impensé des lieux communs de la réflexion québécoise en philosophie, Hébert se fait ethnophilosophe (1983e). Qu'est-ce à dire? Abordant la philosophie comme une discipline constituée, Hébert juxtapose, accumule, démultiplie, dresse une "liste indéfinie de questions " (1983e) sur ses déterminants territoriaux internes et externes, tels que la langue et ses modalités de lire et d'écrire, les institutions de production-diffusion-consommationconsécration, le sol politique, la circulation des idées. L'excès de la 
remise en question pour décoder et trouer les pistes cul-de-sac. Lisez:

Qu'est-ce que le texte du discours philosophique? Comment s'orienter dans la pensée, hors des frontières qui la déterminent? Universalité des adverbes de temps, adverbes de lieu, adverbes de manière (qui fera la philosophie de ce concept linguistique de manière?); universalité des adjectifs et des pronoms essentiellement relatifs - qui inscrivent l'orientation des corps et mettent en relation. Par conséquent, conscience originaire de ce qui circule à ce moment-là ou ici-maintenant sur chaque territoire offert à l'écriture de son problème. Qui signe "je » de sa manière ou le non-je des échanges rationnels qui en délestent l'anecdote? Qui " philosophe » ou dénie son rapport à quelle philosophie dans un même cadre institutionnel - ou autrement? Quel discours de vérité délivre-t-il, proclame-t-il? dans quelle langue? quelles propriétés syntaxiques sautant aux yeux de quels lecteurs disponibles dans l'histoire? Au nom de quelle valeur-Justice, quelle valeurHomme/Femme, quelle valeur-Raison, contre quel autre «je » du même "nom "? À quelle urgence de solution ou dissolution nous convie-t-il dans le champ du savoir, vérifiable par quel travail réel, appuyé par les filons de quelles sciences, quelles littératures? quelles politiques? Comment ce discours philosophique est-il formé, informant; par quels instruments de travail, fabriqué donc sous quelles règles de quel genre (ou nouveau genre également réglé)? sous quel consensus territorial ultime, quelle conformité paradigmatique différée pour quelle recherche originale. Et à quel prix - puisque la pensée se monnaye aussi par les figures économiques du papier, ou se paye par l'oblation d'une vie. Oì, sous quels feux de quelle rampe institutionnelle apparaitelle, et à quel moment (et pas un autre) ce travail se dévoile$\mathrm{t}$-il pour se livrer à la circulation d'un va-et-vient international ou à l'interminable circuit de l'interprétation? quelle communication des consciences alors entre "gens de métier? " (1983e).

Nous savons peu de choses sur le mode de penser des philosophes, sur les interactions sociales d'où surgit telle pulsion réflexive [...] Nous manque encore une science des mécanismes créatifs dans le domaine de la pensée et de l'histoire textuelle de la pensée [...] Comment donc s'élabore le discours philosophique? Comment se réécrit-il, matériellement parlant? Comment estil reçu hors du siècle et du pays, à quelles conditions est-il recevable pour les nouveaux lecteurs qui soudain y découvrent une incitation à mieux comprendre leur propre point de vue? (1984a)

Proposant un schéma ethnophilosophique qui s'applique à tous les territoires de la pensée philosophique (1983e), cette interrogation originaire se veut un retour réflexif sur les lieux qui ont défini et 
définissent encore l'exercice des formes de rationalité en philosophie. $C^{\prime}$ est ainsi que ce questionnement circonscrit trois aspects du territoire (1979): 1) La constitution même de la philosophie, comme figure sociale, à savoir ses pratiques et ses produits qui sont une occupation spécifique du sol; 2) la "circulation internationale ou non" des courants de pensée, leurs lectures et leurs valeurs d'usage; 3) le dynamisme qui relie les deux premiers aspects.

C'est à partir des étapes historiques de cette occupation tridimensionnelle et des conjonctures (littéraire, politique, religieuse) qu'existent des histoires nationales de la philosophie. Enfin, chaque développement national a sa «mythologie fondatrice» qui "a pour fonction de rapatrier une histoire spécifique et de mettre en place, de décider, de prolonger des pratiques institutionnelles qui garantissent en un lieu un style » (1979).

En résumé, cette "pratique ethnophilosophante» se révèle être une démarche pour comprendre pour soi-même ce qu'est la philosophie et pour penser autrement son propre rapport à cette expérience. C'est pourquoi, ce programme-questionnement s'adresse, en tout premier lieu, aux philosophes québécois actuels. En effet, face à leurs déterminations historiques, ils sont plusieurs à les ignorer ou à en faire fi (par haine?) ou encore à les épouser (par exaltation de qui?). Aveugles, méconnaissants ou..., ils ne voient pas non plus que ces déterminants territoriaux ont des effets sur leurs modes de lecture et d'écriture en philosophie. Ils ne s'aperçoivent pas, enfin, qu'ils répètent comme les générations antérieures "un même rapport "vécu" à la pensée avec les mêmes privilèges " (1983f) et le même "langage de l'autorité et de l'autorisation" (1979).

Et pour cause. Comment est-il possible de comprendre ces attitudes, ces comportements, ces conduites, ces habitudes de penser, quand la caution légitimante est si (ré)active? Comment décoder des codes déjà considérés comme universels? Comment critiquer non pas pour juger, mais pour laisser jaillir la liberté de penser par soi-même, quand les voies (voix réflexives) sont déjà tracées? Hébert répond: En requestionnant le chemin parcouru par la pensée. Ainsi, dans ce livre-ci, à partir des parcours (-détours) sur les représentations de l'Autre à propos du Québec, Hébert veut chercher à comprendre et à faire comprendre ces rapports des déterminants territoriaux avec une certaine histoire de la pensée québécoise en philosophie.

\section{La querelle atlantique: l'enclave exotique}

Hébert choisit de présenter en deuxième partie du livre (pp. 41-211) vingt-sept écrits d'observateurs externes - notamment des Français, Anglais, Italiens, Belges et Américains - répartis sur quatre périodes ponctuées par deux moments majeurs dans la Querelle séculaire au sujet du Nouveau Monde. Cette liste ne comporte pas les noms de grands penseurs qui généralement ont sinon boudé l'Amérique française, du moins négligé d'approfondir leurs "intuitions " 
souvent lapidaires présentées dans la première partie du livre sous forme de "Grapho-clips» (pp. 31-40). Quoi qu'il en soit, "au fil des intérêts [...] entre observateurs et observés» (p. 16), ces voix de l'Autre ont élaboré diverses formes d'un discours exotique d'exaltation ou de condamnation au sujet du territoire francophone en Amérique du Nord. Ce discours a pour référence et pour contenu d'une part les déterminants territoriaux des quatre pays colonisateurs - Angleterre, France, Rome, États-Unis - qui sont projetés sur le Québec; d'autre part, la structure philique et phobique telle qu'éprouvée et vécue par l'Amérique française à l'égard de ces mêmes quatre pays (pp. 20-21). Ainsi, il ne s'agit pas d'une simple image de soi-même inventée par Autrui que le Québec aurait intériorisée. Le rapport est dialectique. Mais dans l'état actuel de cette étude, il est difficile, me semble-til, d'en décrire avec précision le processus. Ce corpus-ci montre que l'étranger perçoit l'Amérique française par ses yeux. En d'autres termes, il découvre sous des Québécois sa propre figure. Mais il est bien possible que cette lecture externe de l'Amérique soit aussi la représentation que se fait le Québec de lui-même (p. 15). En effet, tout au long de cette histoire, des "Québécois-Amis" ont donné hospitalité (1983d) au regard de l'Autre sur eux-mêmes et ont participé par leur pratique à l'édification de cette image. Des échanges convenus et réglés, voire des "alliances" (1979) montrent, par exemple, que le visiteur de passage s'est parfois fait le "publiciste" (1989) de ce que l'hôte désirait entendre. À l'inverse, il est arrivé que l'hôte (1984b) ait accepté de transplanter en Amérique des paradigmes étrangers: il s'est fait "l'hôte de l'autre hôte" (1983d) et auteur $d^{\prime}$ une "histoire endocolonisante" (p. 22).

Il en résulte une représentation "imaginaire » du Québec qui a pris diverses formulations. Selon que ce territoire fait partie et participe de l'une ou l'autre de ces puissances euro-américaines ou encore développe des traits de l'une de ces mêmes puissances, mais "à travers des clôtures"(p. 26, note 5), apparaissent les expressions suivantes: "Petite France transatlantique » (p. 18); "pays de mission »; "vestiges de l'Ancien Régime "; "confluent de l'Europe et de l'Amérique »; etc. Finalement toutes ces représentations renvoient aux mythes de la plaque tournante et du carrefour d'influences, aux clichés du cousinage, du voisinage et du sillage. Plus encore, ces jeux d'alliance et de xénophilie peuvent se transformer en mésalliance et en xénophobie. Pour plus de clarté, je présente dans un tableau d'ensemble les diverses formes qu'a prises la fonction exotique de ce Québec considéré comme enclavé: 


\begin{tabular}{|c|c|c|c|c|}
\hline D'UN LIEU & \multicolumn{2}{|c|}{ À UNE GÉOTOPIQUE DE L'ENCLAVE } & & \multirow{2}{*}{$\begin{array}{l}\begin{array}{l}\text { À L'HISTOIRE LOCALE DE } \\
\text { LA PRATIQUE PHILOSOPHIQUE }\end{array} \\
\begin{array}{l}\text { La France impose sa pensée } \\
\text { sur le territoire conquis: } \\
\text { Philosophie scolastique }\end{array}\end{array}$} \\
\hline Nouvelle-France & $(1608-1750)$ & Fonction exotique utopique & & \\
\hline$\hat{A}_{\text {ge politique }}$ & $\begin{array}{l}\text { Période I } \\
\text { (1756-1789) } \\
\text { 1. Mirabeau } \\
\text { 2. Bougainville } \\
\text { 3. Raynal } \\
\text { 4. Burke } \\
\text { 5. Crèvecoeur } \\
\text { 6. Condorcet } \\
\text { 7. Fox }\end{array}$ & $\begin{array}{l}\text { Fonction exotique de } \\
\text { l'interruption } \\
\text { Québec: Être étranger dans } \\
\text { son propre pays qui est libéré, } \\
\text { grâce à la Cession, du } \\
\text { despotisme de l'Ancien } \\
\text { Régime }\end{array}$ & $\begin{array}{l}\text { Moment I de la } \\
\text { Querelle du } \\
\text { Nouveau Monde }\end{array}$ & Philosophie des Lumières \\
\hline $\begin{array}{l}\text { De la guerre de } \\
\text { Sept Ans } \\
\text { (1756-1763) } \\
\text { au Pacte fédératif } \\
\text { à l'ombre de la } \\
\text { guerre civile } \\
\text { américaine }\end{array}$ & $\begin{array}{l}\text { Période II } \\
\text { (1820-1867) } \\
\text { 8. Chateaubriand } \\
\text { 9. Tocqueville } \\
\text { 10. Stuart Mill } \\
\text { 11. Thoreau } \\
\text { 12. Marmier } \\
\text { 13. Rameau }\end{array}$ & $\begin{array}{l}\text { Fonction exotique de } \\
\text { la continuité } \\
\text { Québec: débris-ves tges } \\
\text { de la France ancienne. } \\
\text { "Fonction exotique du frag- } \\
\text { ment et de ses appartenances, } \\
\text { fragment-copie, fragment-com- } \\
\text { pensation rappelant le moment } \\
\text { d'un tout" (p. 18) révolu. }\end{array}$ & & $\begin{array}{l}\text { Philosophies romantiques } \\
\text { (adaptation des modèles } \\
\text { étrangers) }\end{array}$ \\
\hline \multirow[t]{2}{*}{$\begin{array}{l}\text { Âge religieux } \\
\text { Du Pacte fédératif } \\
\text { et de la question } \\
\text { romane à la } \\
\text { Rćvolution } \\
\text { Tranquille }\end{array}$} & $\begin{array}{l}\text { Période III } \\
\text { (1867-1914) } \\
\text { 14. Adams } \\
\text { 15. Léon XIII } \\
\text { 16. Fiske } \\
\text { 17. Du Bled } \\
\text { 18. Rossel } \\
\text { 19. Arnould } \\
\text { 20. Boutroux }\end{array}$ & $\begin{array}{l}\text { Fonctıon exotique de } \\
\text { la survivance } \\
\text { Québec: mandataire de la } \\
\text { France en Amérique; "matière } \\
\text { première exportable» et "la } \\
\text { différence» font partic des } \\
\text { "lieux communs } \\
\text { complémentaires" (p. 19) }\end{array}$ & \multirow[t]{2}{*}{$\begin{array}{l}\text { Moment II de la } \\
\text { Querelle du } \\
\text { Nouveau Monde }\end{array}$} & $\begin{array}{l}\text { Philosophie thomiste } \\
\text { vaticanisée } \\
\text { (transposition normative } \\
\text { de modèles étrangers) }\end{array}$ \\
\hline & $\begin{array}{l}\text { Période lV } \\
\text { (1920-1960) } \\
\text { 21. Prat } \\
\text { 22. Viatte } \\
\text { 23. Gilson } \\
\text { 24. Varagnac } \\
\text { 25. Kolnai } \\
\text { 26. Straram } \\
\text { 27. De Corte }\end{array}$ & $\begin{array}{l}\text { Fonction exotıque triomphale } \\
\text { ou périmée }\end{array}$ & & $\begin{array}{l}\text { Philosophie française } \\
\text { (spiritualiste et réflexive) }\end{array}$ \\
\hline $\begin{array}{l}\text { Áge techno- } \\
\text { culturel }\end{array}$ & $\begin{array}{l}1970 \\
1980\end{array}$ & $\begin{array}{l}\text { Entrée dans la modernité } \\
\text { Ouverture } \\
\text { Priorités et rattrapage }\end{array}$ & $\begin{array}{l}\text { Moment III de la } \\
\text { Querelle du } \\
\text { Nouveau Monde }\end{array}$ & $\begin{array}{l}\text { Phénoménologies } \\
\text { Philosophies anglo-américaines }\end{array}$ \\
\hline
\end{tabular}


Certes, ces idées du territoire sont ici des approximations que des monographies devraient confirmer (p. 20). Il en va de mème de l'analyse-résumé que je fais des représentations exotiques et des pratiques philosophiques correspondantes. L'important est de constater que tout au long de cette "histoire coloniale", le Québec a été vu et s'est vu à la fois comme un lieu maudit et un lieu idyllique, un territoire exécré et un territoire exalté, un espace méprisé et un espace surestimé. Ainsi, pour ne citer qu'un exemple tiré de ce "dossier Hébert", voici les propos du belge De Corte qui voyait, et ce en 1960, le Canada français comme "une sorte d'incarnation historique de l'aristotélisme chrétien »:

Je risquerais donc une prophétie: le Canada français me paraît être destiné à devenir la plaque-tournante entre l'Ancien Monde et le Nouveau Monde, comme la Belgique l'a été et l'est encore entre le monde germanique et le monde latin. Il n'est nullement exclu qu'il soit un jour le lieu entre l'Ordre et le Progrès. (p. 207).

Loin de penser que cette vision exotisée s'arrête en 1960, elle n'est qu'un jalon d'une histoire qui s'éternise jusqu'à nos jours, à travers et par les Michel Serres, Michel Tournier, Cohen-Solal (1986), Lyotard, Derrida (1989). Par ailleurs, ce livre-ci nous en donne la genèse à travers une enquête rétrospective des réflexions philosophiques de ces grandes figures légitimées que sont Condorcet, Tocqueville, Thoreau, Adams, Gilson, Varagnac, etc. Ainsi, depuis 1760 jusqu'à nos jours, existe une structure répétitive et lancinante d'un territoire francophone exotisé.

\section{«Self-Durhamisme"}

Par ailleurs cette stucture a pour effet de déterminer la manière québécoise de penser. En effet, Hébert montre que nos rapports à la philosophie sont marqués par cette histoire coloniale. L'exotisme itératif du territoire a des répercussions continues sur la réflexivité. Dans notre pays francophone, au nord $\mathrm{du} 45^{\mathrm{e}}$ parallèle, où les sources de notre identité sont exogènes, ressurgissent de période en période des philosophes endocolonisateurs qui troquent leur origine pour une philosophie étrangère interprétée comme la garantie de leur universalité (1989).

La communauté philosophique [a] une longue tradition d'adaptation miméo-réactive aux philosophies étrangères - simplifiées hors contexte, décantées hors histoire, habituellement tronquées de leur profondeur bibliographique et territoriale (1989). 
Leur «autophobie» et leur «xénophilie» territoriales fondent leurs paradigmes. Par ailleurs, leurs emprunts de noms propres de la France, de l'Angleterre, de Rome et/ou des États Unis sont moins des adaptations aléatoires et gratuites à des modes intellectuelles externes que des correspondances et des affinités momentanées que l'hôte établit à partir d'une représentation exotisée de son territoire. Par exemple, si le Québec a été, à une période donnée, thomiste, puis existentialiste, il ne s'agit pas d'une adaptation servile à une "mode" romaine - l'Encyclique de Léon XIII - puis française, encore moins d'un rapport direct à une Province cléricale, puis laïcisée, mais plutôt d'un choix d'un mode de penser exogène déterminé par une image exotique de son propre espace. En second lieu, ce choix toujours réactualisé n'est pas toujours une simple imitation servile. Certes, il est arrivé qu'une philosophie étrangère soit présentée et pratiquée comme un savoir normatif et un "exercice unique de la pensée » (1974) qui s'adonne au seul commentaire. Mais on a aussi utilisé des écoles et des courants - romantique, scolastique, marxiste, etc. - pour expliquer, défendre et justifier des savoirs et des pouvoirs ou encore pour les critiquer et les réformer. Encore là, il y a eu des usages particuliers d'une philosophie "doctrinale » immuable qui cautionnait telle ou telle prise de position, mais il est arrivé aussi que ces usages ont donné lieu à des interprétations d'un courant philosophique. D'autres encore, voyant la philosophie comme une nécessité purement théorique au lieu d'un salut, ont considéré impérieux de réfléchir sur des domaines d'objets - langage, épistémologie, éthique et politique - tels qu'ils se pratiquent et se développent en Europe et aux États-Unis.

Bref, par-delà ces choix et ces usages - qui sont encore mal connus -, il existe une tradition redondante en philosophie québécoise qui consiste à se déterminer dans la réflexion en s'inscrivant dans ce qui se fait ailleurs. "Mêmes habitudes de réception " (1989) depuis deux cents ans. Même schème d'imitation, mais qui correspond à une logique historique non interrogée, niée, censurée, répétitive mais masquée par l'institutionnalisation de la philosophie elle-même. En effet, cette pratique philosophique, active à l'excès, répudie constamment l'histoire de sa propre pensée comme si elle ne voulait pas avoir expérimenté ce rapport de dépendance et parce qu'elle voit dans ce passé, un obstacle à son propre avenir dans la pensée. Au dire de Hébert, cet autodénigrement est du "self-durhamisme" (1989).

\section{Un « Nouveau Monde auto-réflexif »}

Face au "stigmate honteux" (1989) de nos origines et des impasses auxquelles conduisent toutes les dénégations, notamment dans nos manières de penser en philosophie, il importe de faire front à ce qui est incontournable. Mais comment? En pratiquant les possibilités de l'ethnophilosophie (1983e) qui permet d'arriver à l'universel par d'autres canaux que celui de l'imitation. En effet, au lieu de tenir pour rien ou - ce qui revient au même - de (sur)valoriser les 
expériences de réflexion qu'ont faites les philosophes - penseurs du Québec, il importe d'une part, de faire l'histoire de cette pensée et d'autre part, de prendre pour objet de réflexion théorique des thèmes territoriaux communs à l'humanité. D'un mot, en poursuivant pour soi, pour ici et pour maintenant les questions et les remises en question de toutes nos déterminations territoriales qui sont contemporaines de la constitution de nos paradigmes philosophiques (1983e), il s'agit de transformer - comme nous l'écrivions plus haut - nos rapports à notre propre histoire et à la philosophie. En d'autres termes, plutôt que de fuir une identité dédoublée, imaginaire, sublimée, aveuglante (1989) par des substituts étrangers issus de d'autres cultures qu' on imite dans l'espoir d'obtenir une "identité - sanction - référence " (1974), il s'agit d'assumer son identité particulière et régionale, sachant qu'elle est, elle aussi, toute l'humanité.

Ainsi, au lieu de raturer cette expérience spécifique et singulière de la réflexion québécoise en philosophie et dans l'histoire de la pensée euro-américaine, il faut développer, selon Hébert, "des schémas de reconnaissance des lieux [...] de la philosophie québécoise [...] normalisés, publiés, contrôlés, acquis [...], ruminés, réfléchis (et) intégrés à tous les départements francophones de philosophie » (p. 10 et 1989). En effet, que cette histoire régionale soit faite de dépendance et d'imitation, il n'en demeure pas moins qu'il s'agit d'une expérience humaine dans la pensée qui attend son explication historique et sa théorie philosophique. Paradoxalement, ce type d'histoire n'est toujours pas écrit. Un bref rappel de l'histoire de cette historiographie philosophique - L.A. Pâquet, A. Robert, H. Bastien, V. Cauchy, Y. Lamonde, R. Houde, J.-P. Brodeur, H. Malouin, M. Chabot, J. Beaudry, J.G. Ruelland, $R$. Hébert, etc. - permet de constater « la langueur de la narration historico-philosophique du corpus québécois » (p. 10). En outre, ces travaux historiographiques ne sont presque jamais interprétés et réévalués tant dans leurs résultats et leurs visées que dans leurs méthodes et leur définition de la réflexion québécoise en philosophie. Il faut lire, "s'entrelire pour oser s'entre-penser » (1983a) et rassembler les monographies. Il faut aussi mettre à l'épreuve cette hypothèse nouvelle proposée par Hébert des déterminations territoriales sur la pensée d'ici. Ainsi, supposant que l'histoire de notre pensée consiste en l'adoption de philosophies exogènes, il faut expliquer pourquoi et comment la réflexion d'ici, eu égard à sa situation, aux circonstances concrètes et aux possibilités, a choisi tel ou tel courant philosophique. Quels en ont été les usages? Est-ce que ces usages ont parfois donné lieu à des interprétations du système philosophique étranger? Faut-il parler de rupture entre telle forme de pensée et telle autre? Les réponses à ces questions feront peut-être découvrir, d'une part, l'existence "d'une manière différente de vivre l'exercise de la pensée » (1974) et d'autre part, au coeur même de l'histoire de cette pratique de la réflexion - certes à dépasser et à transformer - des éléments d'assimilation-accommodation à des exigences et à des limites. Ainsi, résulteront une réévaluation de la tradition théorétique qui nous 
est propre de même que la découverte, malgré toutes les imitations serviles, toutes les répétitions, tous les intellectualismes autoritaires, de nos "habitudes» (1979) dures à chercher par la réflexion, sinon à nous libérer tout à fait, du moins à dire librement cette quête.

\section{Repères bibliographiques}

1974:

"Pensée québécoise et plaisir de la différence », Brèches, $\mathrm{n}^{\circ} 3,31-39$.

1979: “Philosophies, nationalités: pour un traitement géotopique », Bulletin de la Société de Philosophie du Québec, vol. 5, n4, déc., 52-56.

1980: c.r.: «Roland Houde. Histoire et Philosophie au Québec, Anarchéologie du savoir historique, Trois-Rivières, Bien Public, $1979 »$, Philosophiques, vol. VII, n 1, 93-100.

1981: $\quad$ é.c.: «Rationalité-N d'un colloque sur les $\mathrm{N}$. rationalités. Rationality today/La rationalité aujourd'hui. T.G. Geraets (dir.)", Philosophiques, vol. VIII, $\mathrm{n}^{\circ} 1,139-148$.

1982-1983a: é.c.: « D'une falaise d'où l'on voit poindre le soleil de la culture savante. Contribution au premier cahier de l'I.Q.R.C. ", Philosophiques, vol. IX, $\mathrm{n}^{\circ} 2$, oct., 281-293; vol. X, $\mathrm{n}^{\circ} 1$, avril, 97-110.

1983b: $\quad$ c.r.: "Collectif Philosophie et littérature, coll. l’Univers de la philosophie, Montréal, Bellarmin, 1979, 189 p.", Philosophiques, vol. X, n¹, avril, 188-191.

1983c: c.r.: "Lorsque la raison se démarquait de son autre... Platon, les mots et les mythes de Luc Brisson, Maspéro ", Spirales, déc.,13.

1983d: "La philosophie devant l'hôte: hospitalité, ou le contredon des savoirs " dans Objets pour la philosophie, Québec, 1983, 137-149.

1983e: $\quad$ Préface aux civilités frontalières de la pensée, Montréal, Éd. Temora,1983, 20 p. (version manuscrite en 1981).

1983f: "Hypothèses laconiques sur un lieu en temps de paix ", La Nouvelle Barre du Jour, $\mathrm{n}^{\circ} 130-131,113-134$.

1984a: é.c.: “Composantes du laboratoire politique: sur la réception de L'anomalie sauvage: puissance et pouvoir chez Spinoza d'Antonio Négri », Dialogue, XXIII, 315-325.

1984b: c.r.: «Derrida, ombilic de nos limbes? "Spirales, oct., 3.

1984c: $\quad$ é.c.: "Sans trop mâcher les mots, percevoir. Contribution au Réjean Ducharme, Nietzsche et Dionysos de René LeducPark", Philosophiques, vol. XI, n 1, avril, 191-202.

1986: "Perles, prédicats et prédication sartrienne ", Fragments, $n^{\circ} 35-36,1-7$. 
1989: «Fracture endo-coloniale-Autour d'un anniversaire et quelques identités", La Petite Revue de Philosophie, vol. $11, \mathrm{n}^{\circ} 1,63-83$.

1990: c.r.: "Martin Heidegger: Les hymnes de Hölderlin: "La Germanie" et "le Rhin", tr. F. Fédier et J. Hervier, Paris, Gallimard, $1988 »$, Philosopher, n 9, 198-201.

Département de philosophie

Université du Québec à Montréal 